\title{
Nucleon resonances and processes involving strange particles
}

\author{
S. Ceci, A. Švarc and B. Zauner \\ Rudjer Bošković Institute, \\ Bijenička c. 54, \\ 10000 Zagreb, Croatia \\ E-mail: Alfred.Svarc@irb.hr
}

December 14, 2018

\begin{abstract}
An existing single resonance model with S11, P11 and P13 BreitWiegner resonances in the s-channel has been re-applied to the old $\pi \mathrm{N} \rightarrow \mathrm{K} \Lambda$ data. It has been shown that the standard set of resonant parameters fails to reproduce the shape of the differential cross section. The resonance parameter determination has been repeated retaining the most recent knowledge about the nucleon resonances. The extracted set of parameters has confirmed the need for the strong contribution of a $\mathrm{P} 11(1710)$ resonance. The need for any significant contribution of the P13 resonance has been eliminated. Assuming that the Baker. et al data set [1] is a most reliable one, the P11 resonance can not but be quite narrow. It emerges as a good candidate for the non-strange counter partner of the established pentaquark antidecuplet.
\end{abstract}

In spite of the fact that the experimental data for the process $\pi \mathrm{N} \rightarrow \mathrm{K} \Lambda$, which show a distinct peeking around the energy range of $1700 \mathrm{MeV}$, are available for quite some time [1, 2] the existence of a $\mathrm{P}_{11}(1710)$ resonance in that energy range is not generally accepted, but even questioned [3, 4]. However, all coupled channel models [5] do accept the $\mathrm{P}_{11}(1710)$ state as a legal and needed state, and the general agreement is that it is strongly inelastic.

The confirmation and the additional proof for the existence of the $\mathrm{P}_{11}(1710)$ resonance turned out to be critically needed quite recently in light of reported 
observations of exotic pentaquark $\Theta(1539)$ and $\Xi(1862)$ states 6 , as the mentioned state turns out to belong quite naturally into the pentaquark antidecuplet configuration predicted by recent chiral soliton[7] and $q^{4} \bar{q}$ strong color-spin correlated [8] models.

Keeping in mind the accumulated knowledge about nucleon resonances [5] we have applied the existing, single resonance model of $\pi \mathrm{N} \rightarrow \mathrm{K} \Lambda$ process 9 ] to the existing data set [2], and allowed for the explicit presence of a narrow $\mathrm{P}_{11}$ resonance. The single resonance model calculation has been repeated using the standard set of parameters for the S11(1650), P11(1710) and P13(1720) resonances of ref. 10, and they are given in Table 1 denoted as "PDG". As it is shown in Figs.1 and 2. (thin solid line) that choice of parameters reproduces only the absolute value of the total cross section quite well, and manages to reproduce the shape of the angular distribution only at $\mathrm{w}=1683 \mathrm{MeV}$. It fails miserably in reproducing the shape of the differential cross section at other energies. To eliminate the problem we have fitted the $K \Lambda$ branching ratios in the Breit-Wigner parameterization of the afore discussed resonances to the available experimental data set, enforcing a good description of the absolute value of the total cross section simultaneously keeping the shape of the differential cross sections of the $\pi \mathrm{N} \rightarrow \mathrm{K} \Lambda$ process linear in $\cos (\theta)$ (indicated by experimental data of ref.[1]).

The following resonance parameter extraction method has been applied:

we have started with the belief that when a set of resonance parameters (masses, widths and branching fractions) is once established in any analysis using channels other then $K \Lambda$ channel, the only parameter which we are allowed to vary is a branching fraction to $K \Lambda$ channel, while everything else (masses, widths, branching fractions to other channels) can not be changed. Hence, as starting values we have used the resonant parameters for the $S_{11}$, $P_{11}$ and $P_{13}$ resonances obtained in the coupled channel analysis of $\pi$-nucleon scattering based on the $\pi$-elastic and $\pi \mathrm{N} \rightarrow \eta \mathrm{N}$ channels [11. The only parameter which was allowed to vary was the branching fraction to the $K \Lambda$ channel.

Resonance parameters for the single resonance model.

\begin{tabular}{|l|ccccccccccccc|}
\hline & \multicolumn{4}{|c}{$M$} & \multicolumn{4}{c}{$\Gamma[\mathrm{MeV}]$} & \multicolumn{3}{c|}{$x_{\pi N}[\%]$} & \multicolumn{3}{c|}{$x_{K \Lambda}[\%]$} \\
& $S_{11}$ & $P_{11}$ & $P_{13}$ & $S_{11}$ & $P_{11}$ & $P_{13}$ & $S_{11}$ & $P_{11}$ & $P_{13}$ & $S_{11}$ & $P_{11}$ & $P_{13}$ \\
\hline \hline PDG & 1650 & 1710 & 1720 & 150 & 100 & 150 & 70 & 15 & 15 & 7 & 15 & 6.5 \\
Sol 1 & 1652 & 1713 & 1720 & 202 & 180 & 244 & 79 & 22 & 18 & 2.4 & 23 & 0.16 \\
Sol 2 & 1652 & 1713 & 1720 & 202 & 180 & 244 & 79 & 22 & 18 & 2.4 & 35 & 0.16 \\
Sol 3 & 1652 & $\mathbf{1 7 0 0}$ & 1720 & 202 & $\mathbf{6 0}$ & 244 & 79 & 22 & 18 & $\mathbf{4}$ & $\mathbf{3 0}$ & 0.16 \\
\hline
\end{tabular}




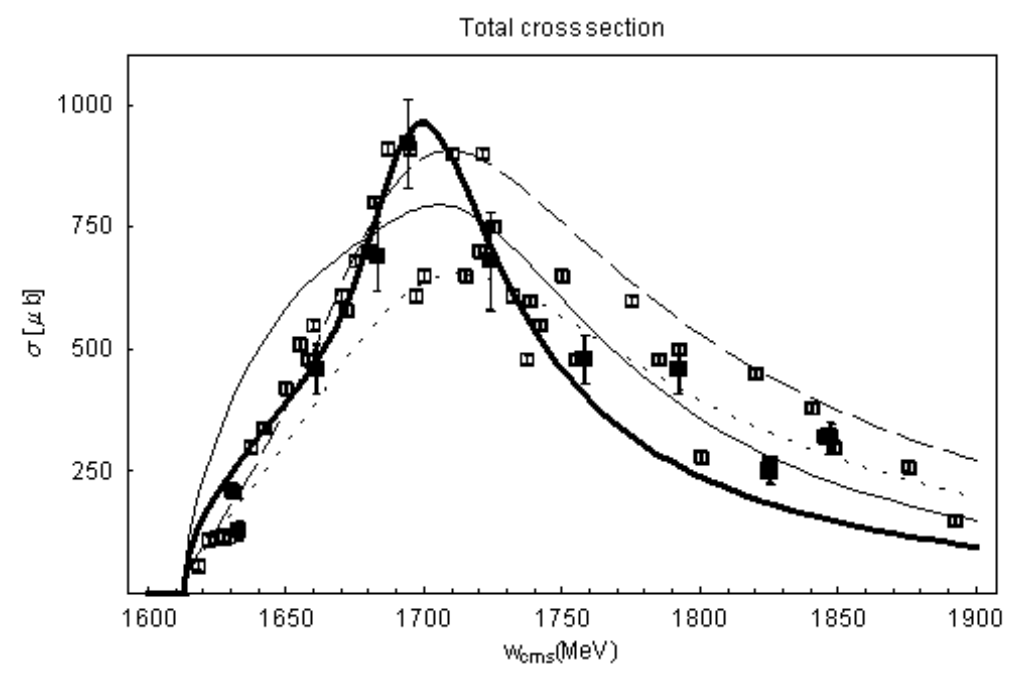

Figure 1: The agreement of the available experimental data for the total cross section (ref.[1] (full boxes); ref.[2]-open boxes) with the single resonance model predictions using different inputs for the resonance parameters: "standard" (PDG) set (thin solid line); Sol 1 (dotted line); Sol 2 (dashed line) and Sol 3 (thick solid line).

The three solutions for the choice of resonant parameters are obtained, and are together with the "standard" (PDG) solution given in Table 1. The agreement with the experimental data is given in Figs. 1. and 2. In extracting new resonance parameters we have kept in mind that the overall data set [1, 2] is mutually inconsistent. In addition, as the latest measured set of data 1] shows a surprisingly narrow width when compared to the overall trend, we have treated it separately with special care.

We fit the "lower" and "upper" band of the total cross section imposing the correct angular dependence at the same time, and obtain Sol 1 (dotted line) and Sol 2 (dashed line). To obtain the Sol 3 (thick solid line) we fit only Baker et al data 1 .

"Standard" (PDG) solution introduces "too much curvature" in the differential cross section throughout the whole energy range indicating too big $\mathrm{P}$-waves contribution relative to the $\mathrm{S}$-wave. If the shape of the angular dependence is to be reproduced, contrary to the standard belief [5, 10], the contribution of $P_{13}$ partial wave is negligible for all obtained solutions. The branching ratio of $S_{11}$ resonance to $K \Lambda$ channel is somewhat smaller then previously believed. The branching ratio of $P_{11}$ resonance to $K \Lambda$ channel is significantly bigger. If the latest Baker et al data [1] are to be taken very 

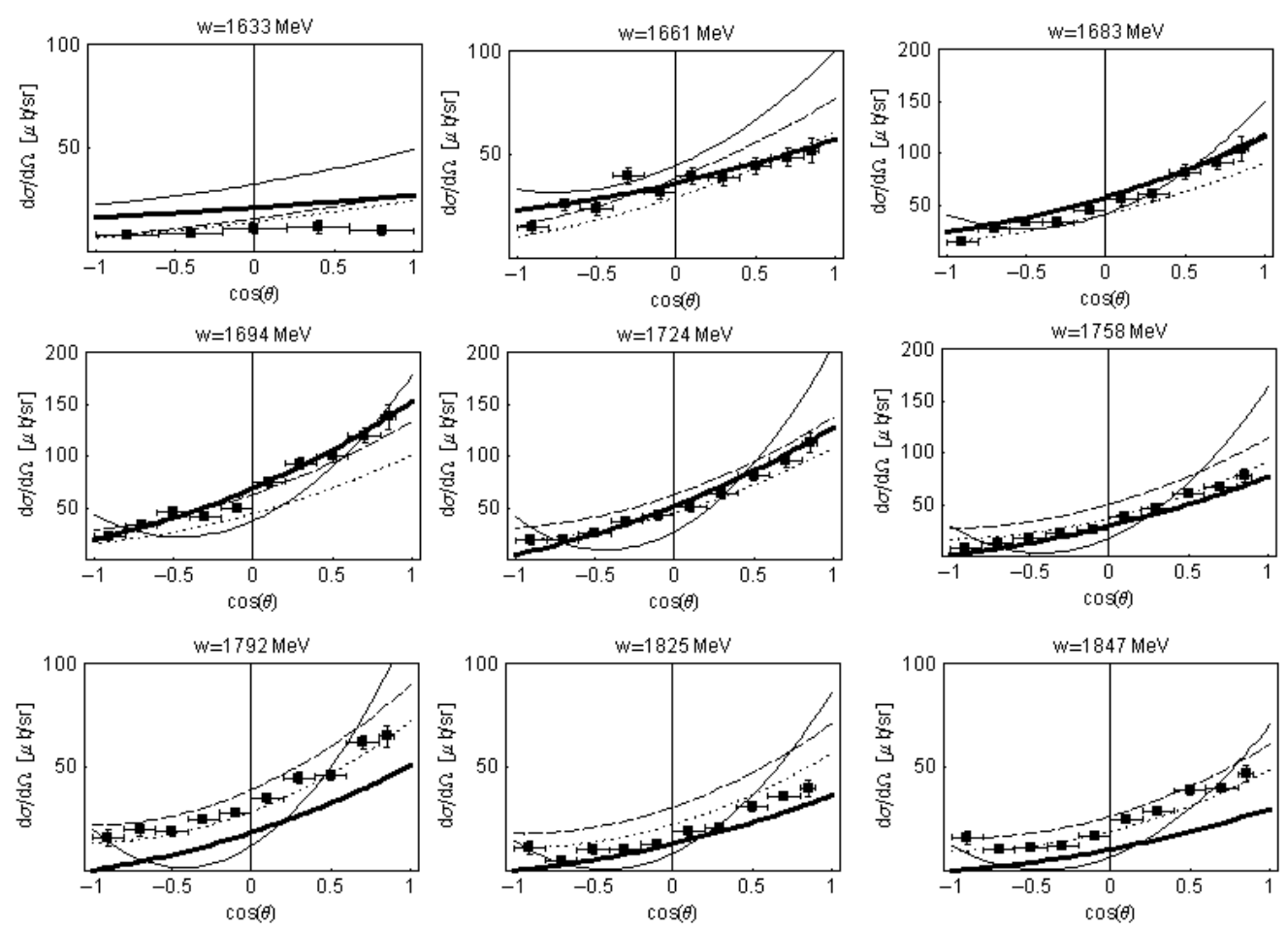

Figure 2: The agreement of the available experimental data for the differential cross section[1] (full boxes) with the single resonance model predictions using different inputs for the resonance parameters: "standard" (PDG) set (thin solid line); Sol 1 (dotted line); Sol 2 (dashed line) and Sol 3 (thick solid line).

seriously the best agreement with the experiment is obtained for very narrow $P_{11}$ resonance, not observed in other processes and strongly inelastic - Sol 3; hence a candidate for a non-strange pentaquark counter-partner.

The re-measuring of the differential cross section for the $\pi \mathrm{N} \rightarrow \mathrm{K} \Lambda$ process in the energy range $1600 \mathrm{MeV}<\mathrm{w}<1800 \mathrm{MeV}$ is badly needed. The decisive conclusion about the existence of the $P_{11}$ non-strange pentaquark counter-partner will be possible only when the improved set of data is fully incorporated in one of the existing coupled channel partial wave analyses $[\mathbf{5}]$.

\section{References}

[1] R.D. Baker et. al., Nucl. Phys. B141, 29 (1978).

[2] Landolt-Börnstein, New Series, ed. H. Schopper, 8 (1973). 
[3] R.A. Arndt, J.M. Ford and L.D. Roper, Phys. Rev. D32, 1085 (1985).

[4] R. A. Arndt et. al., Phys. Rev. C69, 035213 (2004).

[5] R.E. Cutkosky, C.P. Forsyth, R.E. Hendrick and R.L. Kelly, Phys. Rev. D20, 2839 (1979); M. Batinić, I. Šlaus, A. Švarc and B.M.K. Nefkens, Phys. Rev C51, 2310 (1995); T.P. Vrana, S.A. Dytman and T.S.-H- Lee, Phys. Rep. 328, 181 (2000).

[6] T. Nakano et al., Phys. Rev. Lett. 91, 012002 (2003); : NA49 Collaboration, Phys.Rev.Lett. 92, 042003 (2004).

[7] D. Diakonov, V. Petrov and M. Polyakov, Z. Physik A359, 305 (1997).

[8] R. Jaffe and F. Wilczek, Phys. Rev. Lett. 91, 232003 (2003).

[9] K. Tsushima, A. Sibirtsev and A.W. Thomas, Phys.Rev. C62, 064904 (2000).

[10] K. Hagiwara et.al., Phys. Rev. D66, 010001 (2002.)

[11] M. Batinić, I. Dadić, I. Šlaus, A. Švarc, B.M.K. Nefkens and T.S.-H. Lee, Physica Scripta 58, 15 (1998) 